\title{
Single residue in CD28-costimulated CAR-T cells limits long-term persistence and antitumor durability
}

\author{
Sonia Guedan, ${ }^{1,2}$ Aviv Madar, ${ }^{3}$ Victoria Casado-Medrano, ${ }^{4}$ Carolyn Shaw, ${ }^{1}$ Anna Wing, ${ }^{1}$ Fang Liu, ${ }^{1}$ Regina M. Young, \\ Carl H. June, ${ }^{1,5,6}$ and Avery D. Posey Jr. ${ }^{1,5,6,7}$
}

\begin{abstract}
'Center for Cellular Immunotherapies, Perelman School of Medicine at the University of Pennsylvania, Philadelphia, Pennsylvania, USA. 2Department of Hematology, Hospital Clinic, Institut d'Investigacions Biomèdiques August Pi I Sunyer (IDIBAPS), Barcelona, Spain. ${ }^{3}$ Novartis Institutes for Biomedical Research, Cambridge, Massachusetts, USA. ${ }^{4}$ Department of Systems Pharmacology and Translational Therapeutics, University of Pennsylvania, Philadelphia, Pennsylvania, USA. ${ }^{5}$ Department of Pathology and Laboratory Medicine and ${ }^{6}$ Parker Institute for Cellular Immunotherapy, Perelman School of Medicine at the University of Pennsylvania, Philadelphia, Pennsylvania, USA. ${ }^{7}$ Corporal Michael J. Crescenz VA Medical Center, Philadelphia, Pennsylvania, USA.

Chimeric antigen receptor-T (CAR-T) cell therapies can eliminate relapsed and refractory tumors, but the durability of antitumor activity requires in vivo persistence. Differential signaling through the CAR costimulatory domain can alter the T cell metabolism, memory differentiation, and influence long-term persistence. CAR-T cells costimulated with 4-1BB or ICOS persist in xenograft models but those constructed with CD28 exhibit rapid clearance. Here, we show that a single amino acid residue in CD28 drove T cell exhaustion and hindered the persistence of CD28-based CAR-T cells and changing this asparagine to phenylalanine (CD28-YMFM) promoted durable antitumor control. In addition, CD28-YMFM CAR-T cells exhibited reduced T cell differentiation and exhaustion as well as increased skewing toward Th17 cells. Reciprocal modification of ICOS-containing CAR-T cells abolished in vivo persistence and antitumor activity. This finding suggests modifications to the costimulatory domains of CAR-T cells can enable longer persistence and thereby improve antitumor response.
\end{abstract}

\section{Introduction}

Chimeric antigen receptor-T (CAR-T) cell therapies mediate astonishing remissions of hematopoietic malignancies (1-3) and, in a few cases, solid tumors $(4,5)$. However, the antitumor response can be lost due to fleeting persistence of the CAR-T cells $(6,7)$, development of $\mathrm{T}$ cell dysfunction $(8,9)$, or tumor loss of the targeted antigen (10-12). For differences in CAR-T persistence, the prevailing hypothesis has been quantitative signal strength $(13,14)$, as strong $\mathrm{T}$ cell activation potentiates exhaustion (15-17). However, persistence differences can also result from qualitative signaling differences driven by the costimulatory domains of the CAR (CD28, 4-1BB, etc.) (18-22).

Second-generation CD28-based CAR-T cells lack durable persistence in responding patients compared with the persistence observed with 4-1BB-containing CARs. For instance, in a study (NCT02435849) that evaluated 4-1BB-based anti-CD19 CAR-T cells in pediatric acute lymphoblastic leukemia (ALL), the median duration of CAR transgene-positive cells in the peripheral blood of the 60 patients with a favorable response was 168 days (1). Similarly, in a study (NCT02445248) that investigated 4-1BB-based anti-CD19 CAR-T cells in adults with diffuse

\section{Related Commentary: p. 2806}

Conflict of interest: ADP and SG are listed as the inventors on the patent application, W02018140725A1, filed by the Trustees of the University of Pennsylvania and Novartis $\mathrm{Ag}$.

Copyright: (5) 2020, American Society for Clinical Investigation.

Submitted: September 3, 2019; Accepted: February 13, 2020; Published: May 4, 2020.

Reference information: / Clin Invest. 2020;130(6):3087-3097.

https://doi.org/10.1172/JCl133215. large B cell lymphoma, the median duration of CAR transgenepositive cells was 289 days in patients that obtained complete or partial remission and 92 days in all patients (3). However, in a phase I trial of CD28-based anti-CD19 CAR-T cells in adult patients with ALL (NCT01044069), the median duration of CAR-T cell detection was only 14 days (23). Although various other construct differences have been postulated to play a role in in vivo performance and persistence, including the CAR scFv and availability of the target epitope, the CAR costimulatory domain has emerged as the primary predictive factor. The differential persistence of 4-1BB- and CD28-costimulated CARs was first apparent in NSG xenograft models evaluating anti-CD19 and antimesothelin CAR-Ts $(22,24)$. Similarly, the persistence of CD28-based CARs is also dwarfed by the persistence of both 4-1BB- and ICOS-based antimesothelin CARs in xenograft models $(19,25)$. ICOS, a T cell costimulatory receptor of the B7-CD28 superfamily, which also includes inhibitory receptors CTLA-4 and PD-1, shares the signaling motif YMXM with CD28 in its intracellular domain. CARs containing the ICOS costimulatory domain have yet to be evaluated clinically; however, mesothelinspecific ICOS-based CAR-T cells demonstrate robust persistence and antitumor efficacy in xenograft models $(19,25)$.

The shared YMXM motif of CD28 and ICOS, which is well characterized for binding of the $\mathrm{SH} 2$ domain of phosphoinositide 3-kinase, differs only in that $\mathrm{X}$ is asparagine $(\mathrm{N})$ in $\mathrm{CD} 28$ and phenylalanine (F) in ICOS. The asparagine within the YMNM motif of CD28 interacts with Grb2 and is responsible for NFAT activation and IL-2 promoter activity (26). Changing phenylalanine to asparagine in the YMFM motif of ICOS to YMNM induces Grb2 binding, IL-2 production, and NFAT activation upon stimulation of the ICOS receptor. Similarly, mutation of the YMNM motif in CD28 to 
YMAM abolishes VAV1 activation, and reduces PLC $\gamma 1$ activation and calcium influx (27). Robust calcium signaling in T cells is associated with overactivation of NFAT to produce partnerless NFAT, which has been shown to drive T cells into states of exhaustion and dysfunction, specifically through the upregulation of NR4A transcription factors and TOX $(28,29)$. Therefore, we hypothesized that this single amino acid difference in the shared YMXM motif of ICOS and CD28 may explain the difference in persistence of T cells expressing CARs engineered with the intracellular domains from these 2 receptors and changing YMNM to YMFM may promote reduced exhaustion of CD28-costimulated CAR-T cells.

\section{Results}

The persistence of CD28-based CARs can be enhanced through mutation of the Grb2-interacting residue. We used site-directed mutagenesis to generate mesothelin-specific CARs with single amino acid alterations in the YMXM motif - CD28-YMFM and ICOS-YMNM - to complement the existing CD28 (naturally YMNM) and ICOS (naturally YMFM) CARs (Figure 1A). These CARs target mesothelin through the SS1 scFv (30), contain the extracellular spacer region of $\mathrm{CD} 8 \alpha$ (which allows CAR dimerization), transmembrane domains of either CD28 or ICOS, respectively, and the CD3 intracellular domain containing 3 functional immunoreceptor tyrosine-based activation motifs (ITAMs). A first-generation CAR containing only the $\mathrm{CD} 3 \mathrm{z}$ intracellular domain $(\mathrm{z})$ or a truncated form of the CD3z intracellular domain (deltaz) was used as a control as indicated. Expression of the CARs on normal donor T cells was similar among the variant designs (Figure 1B). In vitro characteristics and activities of the variant CD28-CAR-T cell products, including tonic signaling, inhibitory marker expression, and $\mathrm{T}$ cell differentiation, as well as cytotoxicity and cytokine production, were indistinguishable (Figure 1, B-D).

Despite similar in vitro activity, noteworthy differences in CAR-T cell persistence and antitumor activity were detected after treatment of 2 xenograft models bearing well-established subcutaneous or intraperitoneal human pancreatic tumors. Treatment of subcutaneous Capan-2 tumors with either CD28- or CD28-YMFM CAR-T cells exerted a reduction in tumor size for 23 days, at which point tumors treated with CD28-based CAR-T cells resumed growth, while tumors treated with CD28-YMFM-based CAR-T cells maintained durable tumor control (Figure 2, A and B). Tumors treated with ICOS-based CAR-T cells maintained stable disease for 35 days and then initiated a steady reduction in size. Significant differences in $\mathrm{CD}^{+}$and $\mathrm{CD} 8^{+} \mathrm{T}$ cell persistence were observed in the peripheral blood 30 days after CAR-T cell treatment, where CD28-YMFM- and ICOS-based CAR-T cells demonstrated robust persistence and counts of CD28-based CAR-T cells were similar to those of nonspecific, untransduced T cells (Figure 2C). In a model of disseminated pancreatic tumor, ASPC- 1 tumors were established through intraperitoneal injection and treated with control T cells or CD28-, or CD28-YMFM-based CAR-T cells. Similar to the subcutaneous model, CD28- and CD28YMFM CAR-T cells led to tumor reduction for 19 days and disease progression resumed for mice treated with CD28-based CAR-T cells (Figure 2D). Antitumor efficacy continued for CD28-YMFMbased CAR-T cells; eventually, tumor bioluminescence reached background levels of total flux $\left(\sim 1 \times 10^{6} \mathrm{p} / \mathrm{s}\right)$.
Signaling through a CD28-based CAR containing the ICOS YMFM motif shows enhanced AKT phosphorylation with reduced $p-P L C \gamma, p-V A V$, and calcium flux. To understand the mechanism behind the enhanced persistence of CD28, we studied early proximal and distal signaling events after antigen stimulation of mesothelin-specific CAR-T cells, which revealed increases in AKT activation in CD28-YMFM CAR-T cells relative to CD28 CAR-T cells and decreases in VAV1, PLC $\gamma 1$, and ERK activation (Figure 3, $\mathrm{A}-\mathrm{E})$. AKT activation was significantly increased in CD28-YMFM CAR-T cells generated from 4 different healthy donors compared with CD28 CAR-T cells produced from the same donors (Figure 3, D and E). Stimulation of endogenous ICOS has been shown to provide a stronger AKT activation than CD28 signaling through the recruitment of the PI3K regulatory subunit $\mathrm{p} 50 \alpha$, rather than p85 $\alpha$, to the plasma membrane (31). A decrease in VAV1 phosphorylation was expected, as activation of VAV1 by CD28 signaling requires Grb2 binding (32). Once activated, VAV1 signaling leads to NFAT activation and IL-2 production, as well as calcium release through PLC $\gamma 1$ and ERK activation (33). These signaling differences are consistent with the activity expected from altering the Grb2-binding domain of CD28. Additionally, we have previously demonstrated the detection of calcium release from CD28-based CAR-T cells when stimulated with cognate antigen (34) and now show that this calcium response is abrogated when CD28-YMFM CAR-T cells are stimulated by mesothelin; yet, all T cells released calcium in response to TCR stimulation with OKT3 and the calcium ionophore ionomycin (Figure 3F). Taken together, the observed increase in AKT activation and the loss of VAV1 phosphorylation and its downstream cascade of signaling events after stimulation of CD28-YMFM CAR-T cells suggest an alteration of the $\mathrm{T}$ cell signaling that differs from simply an attenuation of signal strength.

In vivo long-term signaling through 28z-YMFM CAR is associated with a transcriptional profile that resembles ICOS signaling. Initial signaling events demonstrated qualitative differences in signaling, as expected from the literature. However, transcriptome analysis by RNA sequencing (RNA-seq) of CAR-T cells 6 days after in vitro antigen recognition revealed that CD28-YMFM CAR-T cells had only 13 differentially expressed genes (DEGs) $(<2$ - or $>2$-fold change) compared with CD28 CAR-T cells (Figure 1E). By contrast, there were 2173 DEGs when comparing ICOS and CD28 CAR-T cells. Thus, CD28 and CD28-YMFM CAR-T cell activities were unremarkable and significant phenotypic differences in persistence and antitumor activity only existed in vivo. Therefore, we established an in vivo model of chronic antigenic stimulation with subcutaneous Capan-2 tumors and harvested T cells from tumors 1 week and 2 weeks after treatment to assess gene expression. Analysis of $\mathrm{T}$ cell infiltration at early time points after in vivo administration (day 7 after treatment) demonstrated that all animals had a similar percentage of $\mathrm{T}$ cells infiltrating the tumor (Supplemental Figure 1; supplemental material available online with this article; https://doi.org/10.1172/JCI133215DS1). Tumor measurements at 2 weeks demonstrated a reduction in tumor growth in all CAR-T cell-treated groups (Figure 4A), but Ki67 expression on CD45+ cells showed that only CD28-YMFM and ICOS CAR-T cells were actively proliferating 2 weeks after treatment (Figure 4B), suggesting that CD28 CAR-T cells had become dysfunctional or inactive. 
A

\begin{tabular}{l} 
ICD8 $\alpha$ leader $\square$ CD28 TM $\square I \operatorname{COS}$ TM \\
$\square$ CD8 $\alpha$ hinge \\
\hline CD28 TM
\end{tabular}

\begin{tabular}{|c|c|c|c|}
\hline deltaz- & SS1 scFv & & \\
\hline & SS1 scFv & CD3 & \\
\hline CD28z & SS1 scFv & CD28 & CD35 \\
\hline CD28(YMFM) & SS1 scFv & CD28-YMFN & CD3द \\
\hline ICOSz & SS1 scFv & HICOS & CD3द \\
\hline ICOS(YMNM)z & $\overline{\text { SS1 scFv }}$ & ICOS-YMNM & CD3६ \\
\hline
\end{tabular}

CD28 RSKRSRLLHSDYMNMTPRRPGPTRKHYQPYAPPRDFAAYRS CD28(YMFM) RSKRSRLLHSDYMFMTPRRPGPTRKHYQPYAPPRDFAAYRS ICOS KYSSSVHDPNGEYMFMRAVNTAKKSRLTDVT ICOS(YMNM) KYSSSVHDPNGEYMNMRAVNTAKKSRLTDVT
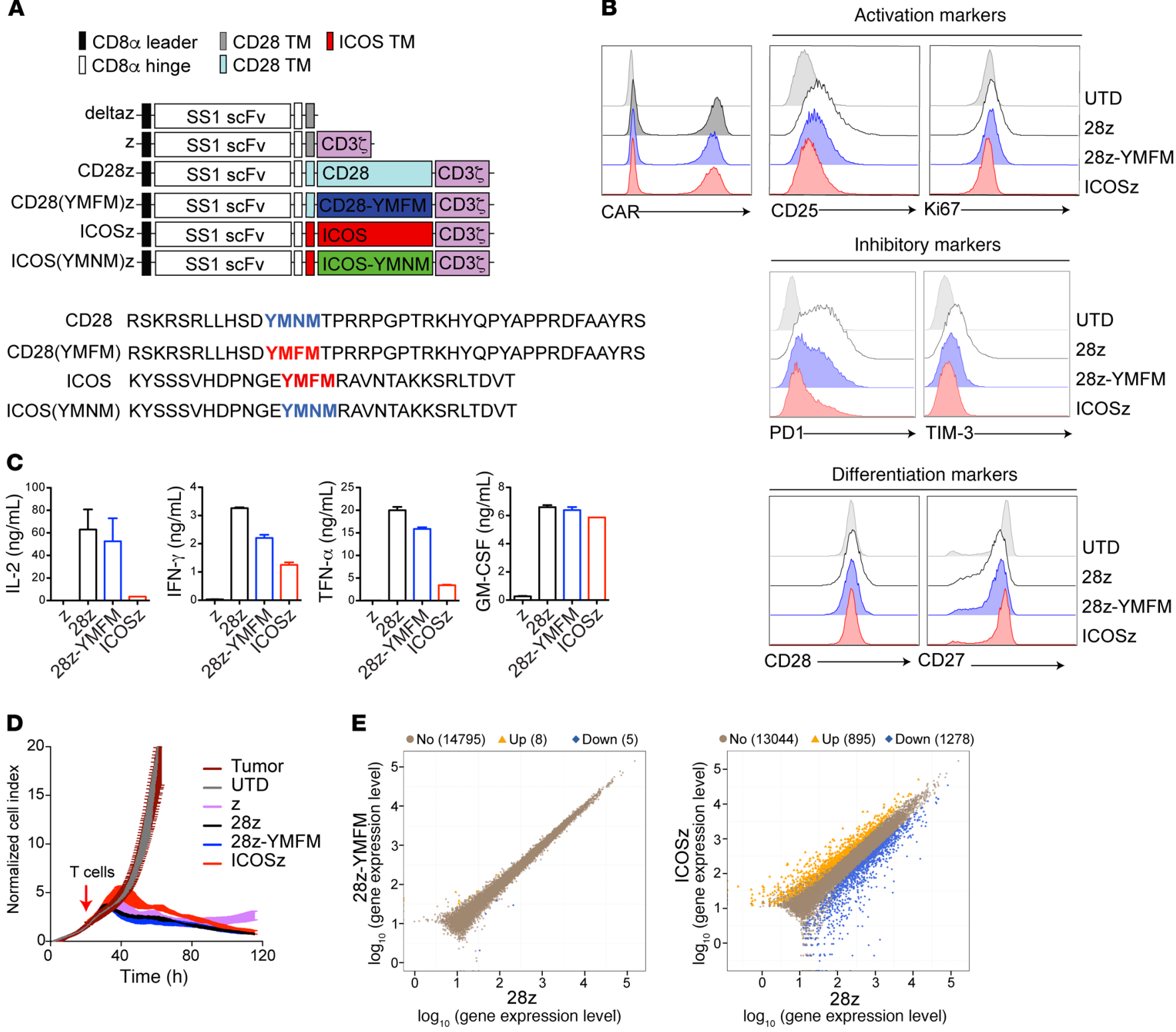
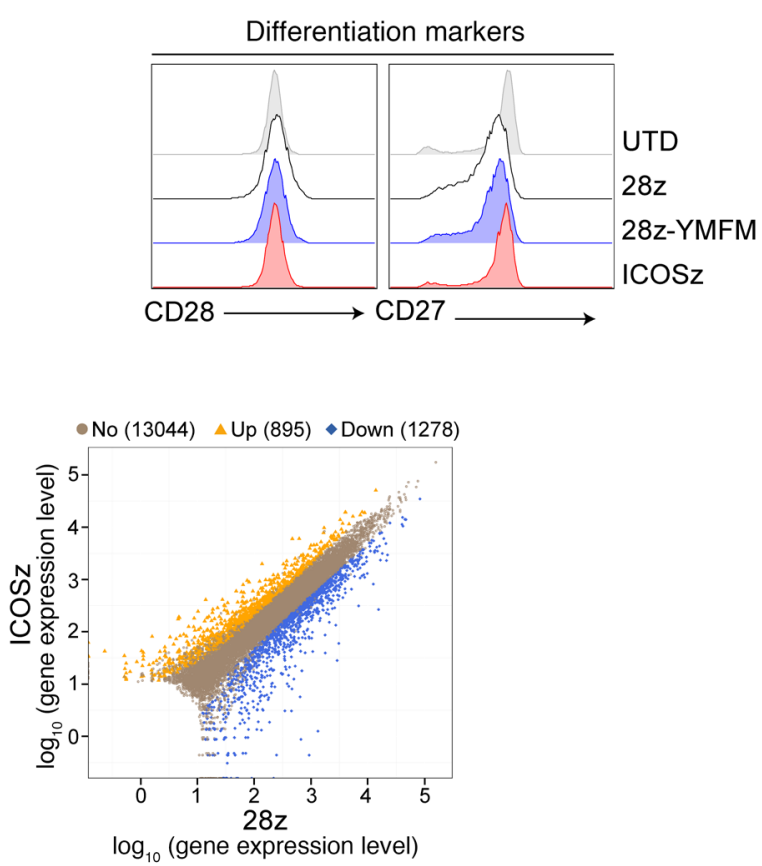

Figure 1. Short-term signaling through 28z-YMFM CAR closely resembles CD28 signaling. (A) Upper panel: Schematic representation of a panel of chimeric receptors that contain the SS1 scFv and differ in the intracellular domain. The CD28(YMFM)z mutant contains the CD28 transmembrane and intracellular domain with a point mutation in the YMNM motif (the asparagine $[\mathrm{N}]$ is mutated to phenylalanine [F]). The ICOS(YMNM)z mutant contains the ICOS transmembrane and intracellular domains with a point mutation in the YMFM motif [the phenylalanine [F] is mutated to an asparagine [N]). Lower panel: The full amino acid sequences for CD28 and ICOS intracellular domains and their corresponding mutants are shown. (B) Characterization of tonic signaling in CAR-T cells during primary expansion. Representative histograms showing the expression of the CAR protein and markers of activation, inhibition, and differentiation in CAR-T cells at 14 days after stimulation with anti-CD3/CD28 beads. (C) CAR-T cells were cocultured with mesothelin+ Capan-2 cells. Supernatants were obtained 24 hours later, and cytokine production was analyzed by Luminex. Representative of 2 donors. (D) A real-time cytotoxicity assay (xCELLigence) was used to evaluate the lysis of Capan-2 tumor cells when treated with CAR-T cells (E/T = 3:1) over a 120-hour period. (E) CAR-T cells from 2 different healthy donors were stimulated with immobilized recombinant mesothelin. Transcriptome analysis by RNA-sequencing was performed 6 days following antigen recognition. Scatter plots show the gene expression levels in 28z-YMFM or ICOSz CAR-T cells versus $28 z$ CAR-T cells. The number of upregulated (orange) and downregulated (blue) genes is indicated.

Gene expression analysis of CAR-T cells 2 weeks after treatment showed clustering of CD28-YMFM with ICOS CAR-T cells, whereas the CD28 CAR-T cells were less related (Figure 4C). These differences were pronounced only after 14 days of stimulation (Figure 4D), with 424 DEGs between CD28 and CD28-YMFM CAR-T cells, 604 DEGs between CD28 and ICOS CAR-T cells, and only 64 DEGs between CD28-YMFM and ICOS CAR-T cells, but few
DEGs after 7 days (Supplemental Tables 1 and 2). We next compared the DEGs to those previously associated with $\mathrm{T}$ cell differentiation and exhaustion. Genes associated with central memory and T memory stem cell (Tscm) differentiation, such as Tcf7, Fosb, and Znf513, were more highly expressed in CD28-YMFM CAR-T cells than in CD28 CAR-T cells (Figure 4E), and the interferon-induced genes Batf2, Cxorf2, and Trim14 were more highly expressed in 
A
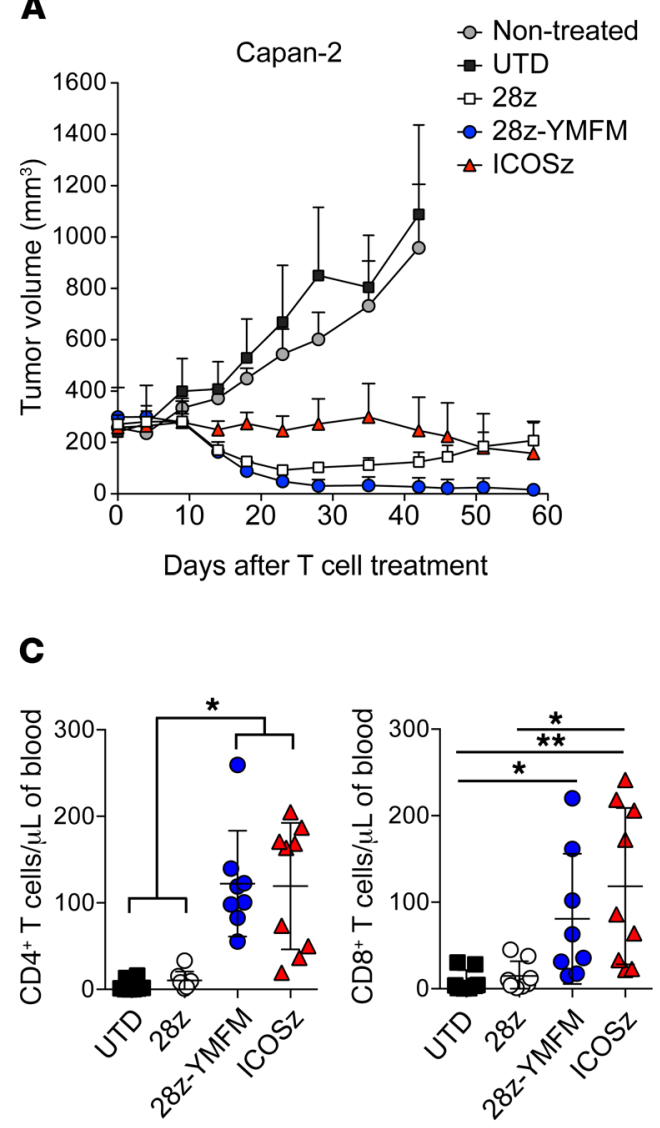

B
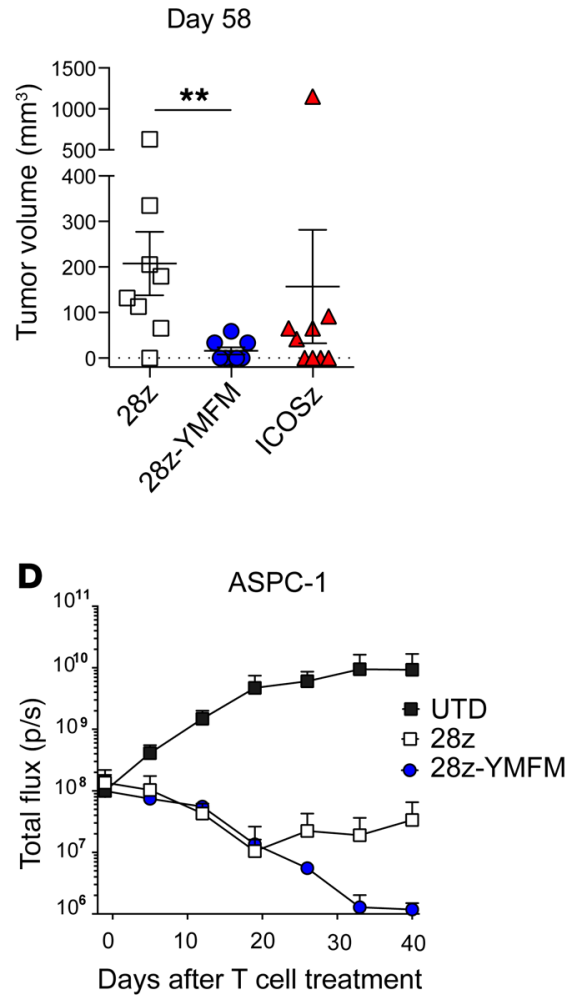

Figure 2. The persistence of CD28based CARs can be enhanced through mutation of the Grb2-interacting residue. ( $A$ and $\mathbf{B}$ ) NSC mice bearing s.c. Capan- 2 tumors were treated 20 days after tumor implantation with 2 doses of control T cells (untransduced, UTD) or CAR-T cells ( $n=8-9$ ) on day 0 and day 15. (A) Tumor volume was analyzed at indicated time points. (B) Tumor volume on day 58 for individual animals is plotted. Error bars represent \pm SEM ( $n=8$ tumors). ${ }^{* *} P<0.01$ by KruskalWallis multiple-comparisons test. (C) The concentration of $\mathrm{CD}^{+}$and $\mathrm{CD} 8^{+} \mathrm{T}$ cells was determined in the blood of treated animals 30 days after $T$ cell injection. Error bars represent \pm SEM $(n=8-9) .{ }^{*} P<0.05 ;{ }^{* *} P<0.01$ by Kruskal-Wallis multiple-comparisons test. (D) NSG mice bearing intraperitoneal $\mathrm{CBG}^{+} \mathrm{ASPC}-1$ tumors were treated 7 days after tumor implantation with $10 \times 10^{6}$ CAR-T cells. Bioluminescence was analyzed at the indicated time points $(n=4)$. both CD28-YMFM and ICOS CAR-T cells than in CD28 CAR-T cells. Similarly, Pik3cd and Dusp6, genes associated with reduced exhaustion or downregulated in effector cells, were more highly expressed in CD28-YMFM CAR-T cells compared with CD28 or ICOS CAR-T cells. $L x n$, which is upregulated in effector cells, had higher expression in CD28 CAR-T cells than CD28-YMFM or ICOS CAR-T cells. Consistently, gene set enrichment analysis (GSEA) revealed upregulation of exhaustion and differentiation pathways in CD28 CAR-T cells, while CD28-YMFM and ICOS CAR-T cells maintained a phenotype of less differentiated $T$ cells (Figure 4F and Supplemental Figure 2). Interestingly, signaling through CD28-YMFM and ICOS polarized CAR-T cells toward a Th17 phenotype, supporting previous results showing that ICOS signaling has a key role in the maintenance of Th17 phenotype and function (Figure $4 \mathrm{G}$ and Supplemental Figure 2).

Mutation of the YMFM motif in the ICOS signaling domain to YMNM (CD28 motif) impairs antitumor effect and T cell persistence. The CD28 intracellular signaling domain contains other important signaling motifs besides the YMNM motif and it is important to study the contribution the Grb2-binding asparagine of the YMNM motif makes to the lack of CAR-T persistence in the absence of these other motifs. Thus, we used a reciprocal CAR design, changing phenylalanine to asparagine in the YMFM motif of the ICOSbased CAR to generate the ICOS-YMNM CAR-T cells (Figure 1A). In vitro characterization of ICOS and ICOS-YMNM CAR-T cell cytotoxicity and cytokine secretion demonstrated no significant differences, as observed with in vitro characterization of the wild- type and variant CD28-based CAR-T cells (Figure 5, A and B). However, treatment of a xenograft model with subcutaneous Capan-2 tumors revealed differences in antitumor activity and robust differences in CAR-T cell persistence (Figure 5, C-E). In this model, ICOS-based CAR-T cells shrink the tumor size, whereas ICOSYMNM CAR-T cells only stabilize the disease. By 36 days after treatment, the persistence of ICOS CAR-T cells dramatically dwarfed that of ICOS-YMNM CAR-T cells, which resembles the persistence of first-generation $\mathrm{CD} 3 \zeta$-only CAR-T cells (Figure 5E). These results demonstrate reciprocal effects of the Grb2-binding residue within the YMXM motif and highlight the Grb2/VAV signaling pathways as targets to enhance CAR-T cell persistence in vivo.

\section{Discussion}

The persistence of CAR-T cells is tightly correlated to the durability of clinical remissions in patients with chronic lymphocytic leukemia (CLL) (7) and ALL (1). Persistence is perhaps even more critical for the clinical success of CAR-T cells in solid tumors, due to a multitude of immunosuppressive obstacles to overcome, the need to eliminate micrometastatic niches, as well as the kinetics required to eradicate significant masses of tumor. Substantial effort has been invested in developing novel approaches to enhance persistence and expansion of adoptively transferred CAR-T cells (35), including selection of specific $\mathrm{T}$ cell subsets (36-38), optimized T cell culture conditions $(39,40)$, combination therapies $(41,42)$, genetic modifications and alternative methods of integration to reduce tonic signaling $(43,44)$, introduction of 

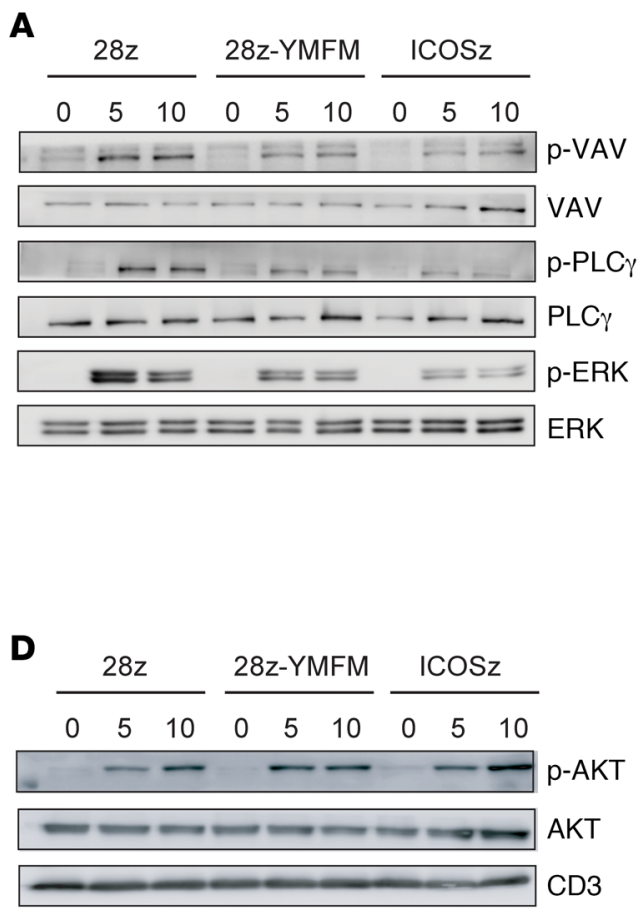

$\mathbf{F}$
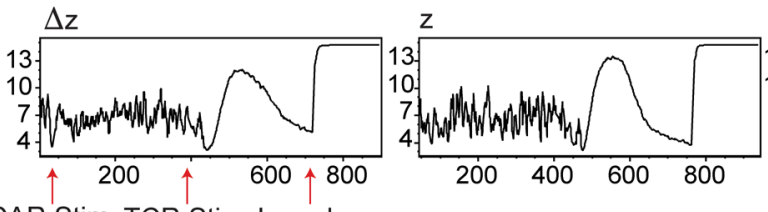

B
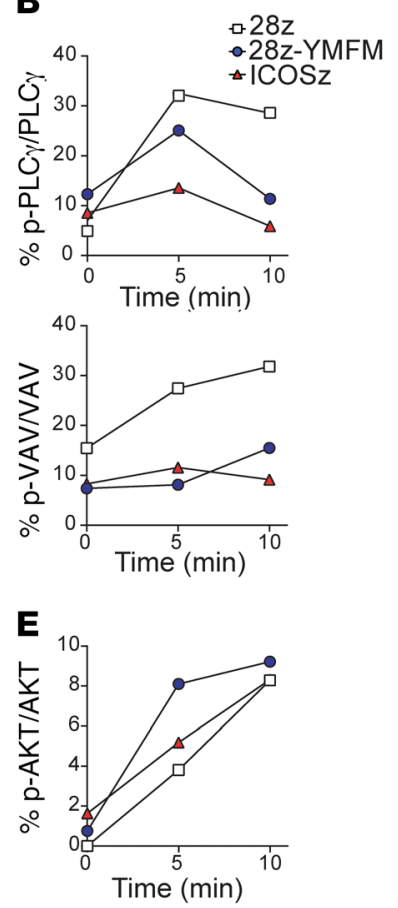

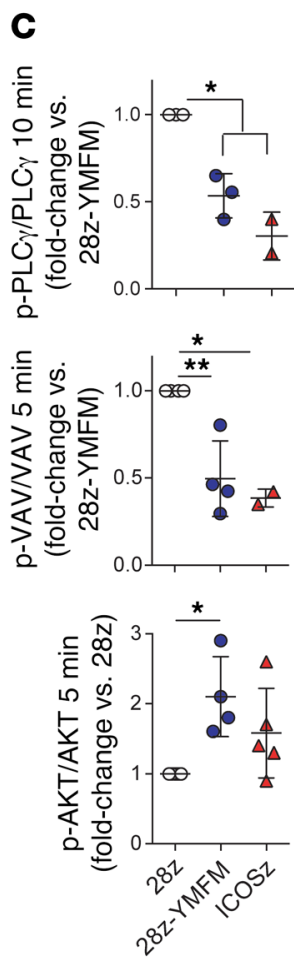

$\mathrm{BBz}$

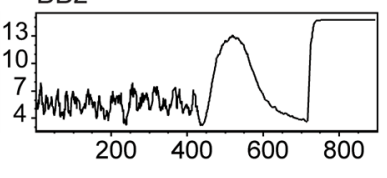

CAR Stim TCR Stim lonophore
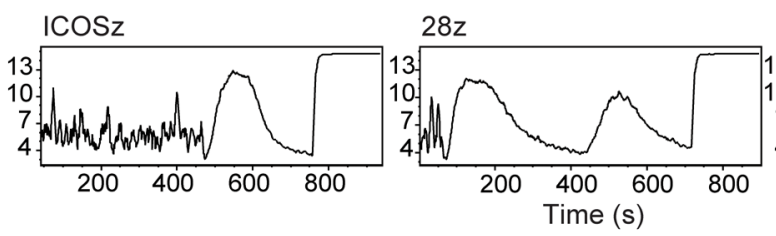

28z-YMFM

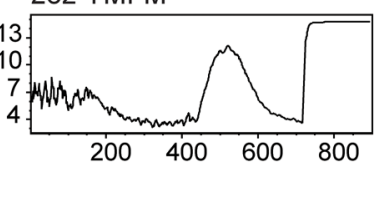

Figure 3. Signaling through a CD28-based CAR containing the ICOS YMFM motif shows enhanced AKT phosphorylation with reduced p-PLC $\gamma$, p-VAV, and calcium flux. (A-E) CAR-T cells were stimulated with magnetic beads coated with recombinant mesothelin. Cell lysates were obtained at 5 and 10 minutes following antigen encounter and phosphorylation levels for VAV, PLC $\gamma$, ERK, and AKT were analyzed by Western blot (A and $\mathbf{D})$ and densitometry (B and E). Basal phosphorylation was evaluated without stimulation (minute 0). (C) T cells from 2 to 5 different healthy donors were stimulated as in $\mathbf{A}$-E, and AKT, VAV, and PLC $\gamma$ phosphorylation was analyzed by densitometry. The mean \pm SD from 4 independent experiments is shown. ${ }^{*} P<0.05$; ${ }^{*} P<0.01$ by 1-way ANOVA with Tukey's post hoc test. (F) Calcium influx was measured in CAR-T cells at baseline for 30 seconds, and then after stimulation with mesothelin-coated magnetic beads for 6 minutes, followed by stimulation with biotinylated OKT3 and avidin for 6 minutes, and treated with ionomycin. The mean Indo-1 ratio of violet/blue fluorescence emission is displayed on the $y$ axis and the time of sample collection in seconds is displayed on the $x$ axis. Representative of 3 different experiments using 3 different normal donors.

ligands for costimulatory molecules (21), providing cytokine signaling (45-49), and the prevention of inhibitory signals $(50,51)$.

Here, we show that persistence and antitumor efficacy of CD28-based CARs targeting mesothelin can be improved by mutating a single amino acid. Because this point mutation does not increase the length of the genetic material to be included in viral vectors, this strategy can be easily combined with other genetic modifications to increase the CAR-T cell therapeutic index. In the pancreatic cancer xenograft models presented here, loss of $\mathrm{T}$ cell persistence leads to tumor outgrowth. However, through the YMFM alteration within the CD28 signaling domain, CAR-T cells demonstrate robust persistence in vivo and durable tumor control. The asparagine in the endogenous CD28 signaling domain interacts with the SH2 domain of Grb2 (52) and this interaction is disrupted through the YMFM alteration (53). This decreased interaction increases AKT activation, reduces the activation of VAV1 and PLC $\gamma 1$, and reduces calcium influx in CAR-T cells stimulated with cognate antigen. These signaling alterations led to significant transcriptional changes that influence reduced $\mathrm{T}$ cell differentiation as well as protection from exhaustion. Importantly, the reciprocal alteration to ICOS with the YMNM motif abolishes $\mathrm{T}$ cell persistence and antitumor activity, highlighting the influence this single amino acid has over control of CAR-T cell persistence and durable antitumor activity. 
A

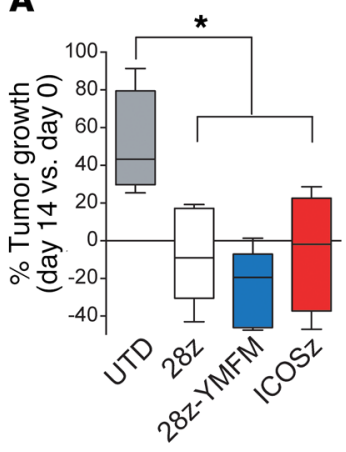

B

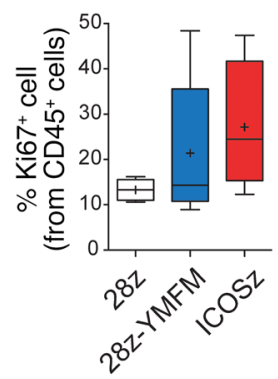

C

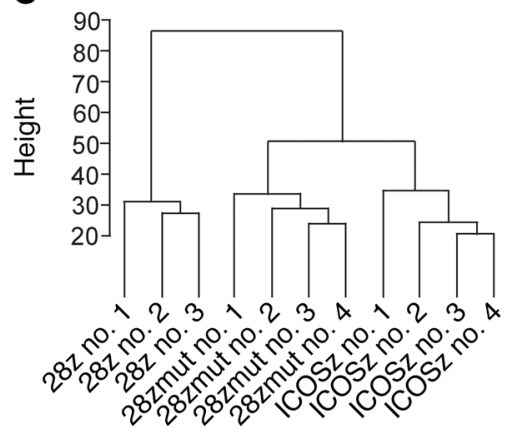

D

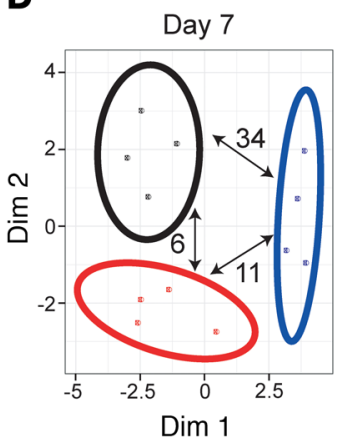

$\mathbf{F}$
E

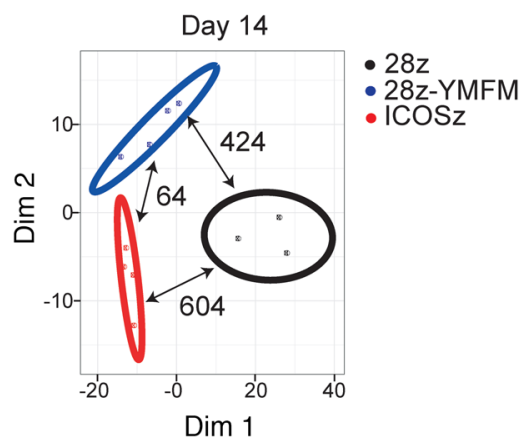

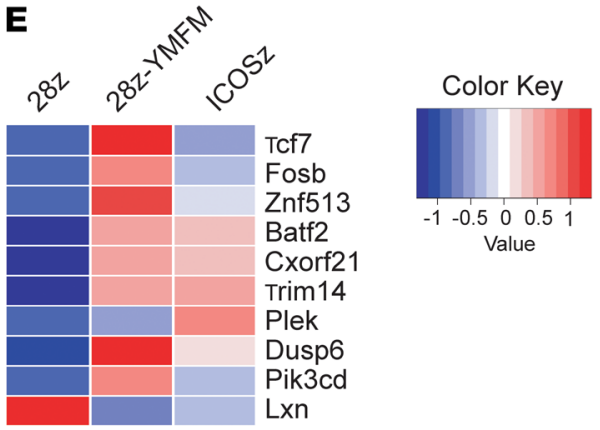

G

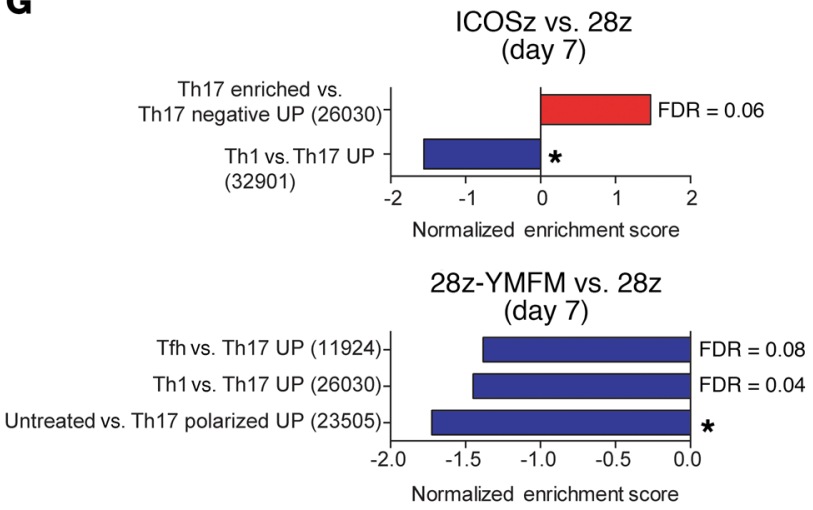

28z-YMFM vs. $28 z$

(day 14)

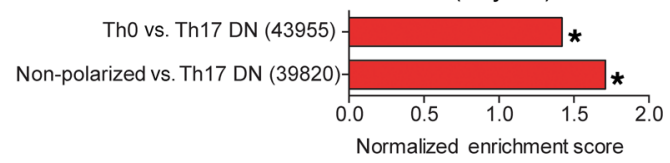

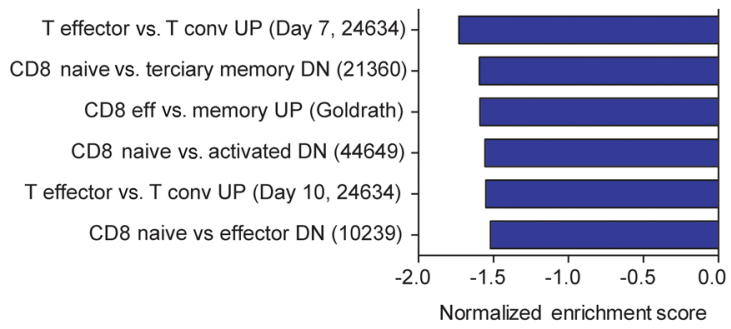

Figure 4. In vivo long-term signaling through 28z-YMFM CAR is associated with a transcriptional profile that resembles ICOS signaling. NSG mice bearing s.c. Capan-2 tumors were treated with CAR-T cells and tumor growth was monitored. T cells were isolated from tumors on day 7 and 14 after treatment for analysis. (A) Box plots of the change in tumor volume on day 14 versus baseline $(n=4-5) .{ }^{*} P<0.05$ by 1 -way ANOVA with Tukey's post hoc test. (B) The expression of CD45 and Ki67 on T cells isolated on day 14 after treatment was analyzed by flow cytometry. For $\mathbf{A}$ and B, box plots show median (line), mean (plus symbol), and 25th to 75th percentile (box). The end of the whiskers represents the minimum and the maximum of all of the data. Error bars represent \pm SEM $(n=4-5) . P>0.05$. (C) Dendrogram showing the relatedness of gene expression patterns for individual animals. (D) MDS analysis of differentially expressed genes between the 3 sets of CAR-T cells isolated from Capan-2 xenograft tumors $(n=3-4)$ on day 7 and day 14 . The number of differentially expressed genes ( $F D R<0.05, F C<2$ or FC $>2$ ) between a pair of CARs is indicated with pair-connecting arrows. (E) Heatmap showing differential expression of genes associated with $\mathrm{T}$ cell differentiation and exhaustion. Genes reported to be preferentially expressed in early stages of $\mathrm{T}$ cell differentiation (Tcf7, Fosb, Znf513, Batf2, Cxorf2, Trim14) and genes upregulated in effector cells (Lxn) or downregulated in effector or exhausted T cells (Dusp6 and Pik3cd) are plotted. (F and G) Normalized enrichment scores of selected up- or downregulated gene sets associated with T cell differentiation (F) or Th17 polarization (C) as determined by GSEA using MSigDB C7 gene ontology sets. ${ }^{*} P<0.05$. For all pathways, FDR $q \leq 0.02$ (unless otherwise indicated). Reference numbers for immunology-related gene lists from MSigDB C7 are indicated in parentheses. 
A

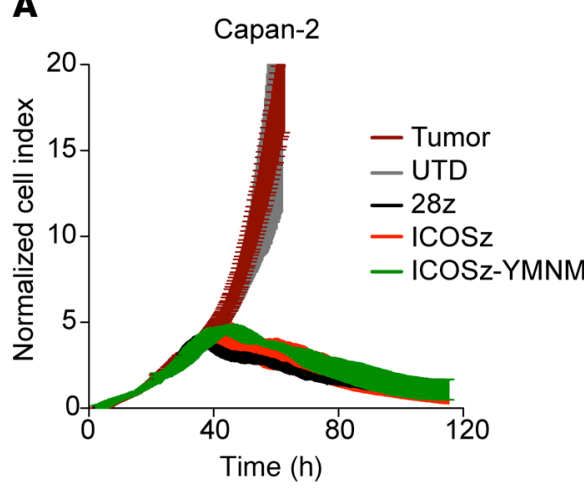

C

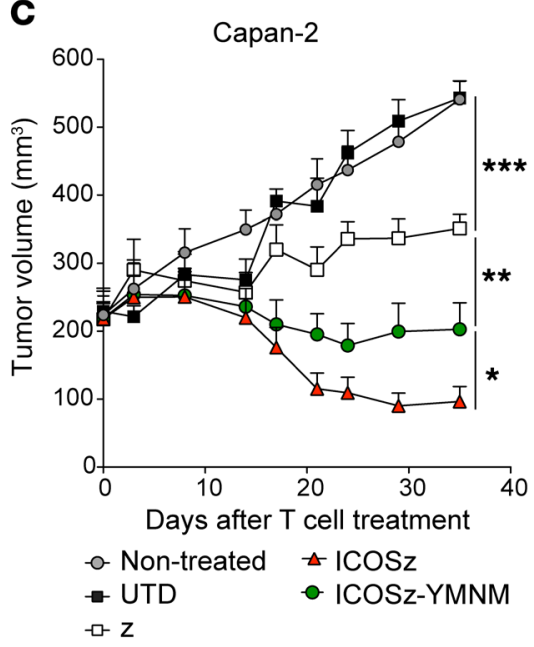

B
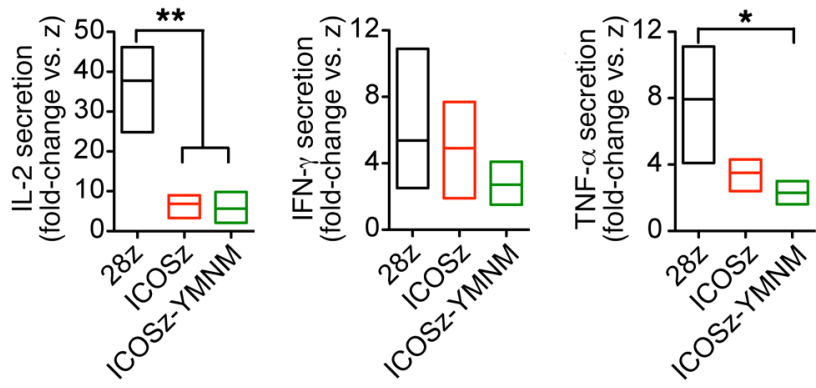

D

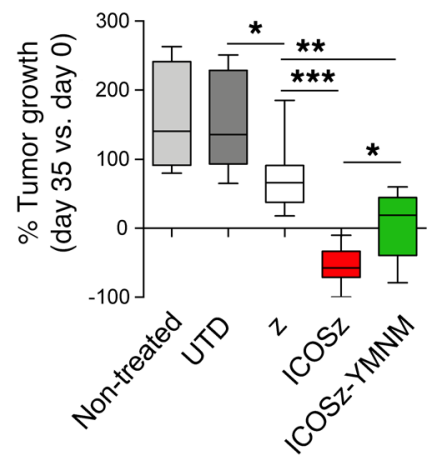

E

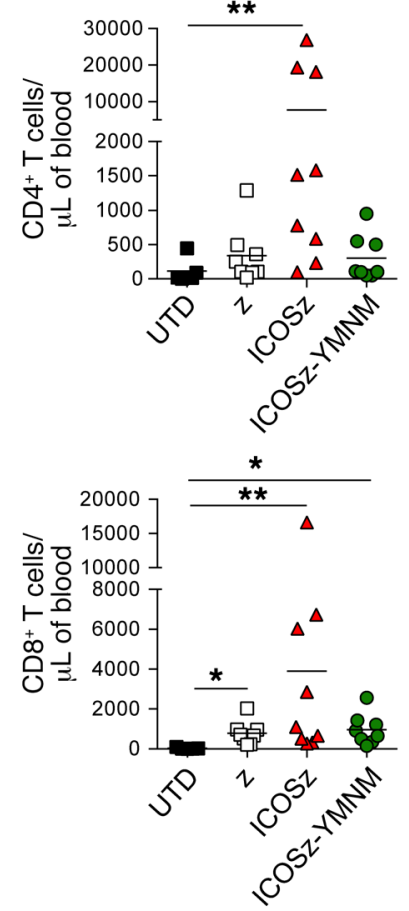

Figure 5. Mutation of the YMFM motif in the ICOS signaling domain to YMNM (CD28 motif) impairs antitumor effect and T cell persistence. (A) A real-time cytotoxicity assay (xCELLigence) was used to evaluate the lysis of Capan-2 tumor cells when treated with CAR-T cells (E/T = 3:1) over a 120-hour period. (B) CAR-T cells were cocultured with mesothelin+ Capan-2 cells. Supernatants were obtained 24 hours later, and cytokine production was analyzed by ELISA. Fold-change cytokine production in second-generation versus first-generation (z) CAR-T cells was analyzed in 3 to 4 healthy donors. $P$ values were calculated by 1-way ANOVA with Tukey's post hoc test. (C-E) NSC mice bearing s.c. Capan-2 tumors were treated 30 days after tumor implantation with 2 doses of CAR-T cells $(n=6-9)$. (C) Tumor volume was analyzed at indicated time points. Statistical analysis was performed by 2 -way ANOVA followed by Dunnett's multiple-comparisons test. (D) Box plots of the change in tumor volume on day 35 versus baseline $(n=6-9)$. Box plots show median (line) and 25th to 75th percentile (box). The end of the whiskers represents the minimum and the maximum of all of the data. $P$ values were calculated with 2-tailed Mann-Whitney tests. (E) The concentration of $\mathrm{CD} 4^{+}$and $\mathrm{CD} 8^{+} \mathrm{T}$ cells was determined in the blood of treated animals 36 days after T cell injection. $P$ values were calculated by Kruskal-Wallis multiple-comparisons test. ${ }^{*} P<0.05$; ${ }^{* *} P<0.01 ;{ }^{* *} P<0.001$.

Alterations to the PYAPP motif within the CD28 costimulatory domain of CARs has been previously reported and was shown to decrease IL-2 secretion and Treg recruitment, reportedly through the elimination of CD28-Lck interactions (54). However, the CD28 PYAPP motif not only interacts with the SH3 domain of Lck (55), but also Filamin-A (56) as well as the SH3 domain of Grb2 $(53,57)$. In fact, the interaction with the Grb2 SH3 domain at the PYAPP motif is thought to reinforce the Grb2 SH2 domain interaction at the YMNM motif (53). T cell persistence was not evaluated in the prior report of $\mathrm{CD} 28$ alteration and it is unclear whether this alteration would enhance antitumor durability, especially since tumor growth was reduced but not eliminated in this model. However, it should be noted that ICOS-costimulated CAR-T cells were not as cytolytic in vivo as CD28-YMFM CAR-T cells and signaling interactions at motifs outside of the YMNM motif in CD28, such as the PRRP and PYAPP motifs, which are responsible for NF- $\mathrm{\kappa B}$ activation and Gads binding (58), may play an important role in this difference.

The decrease in VAV1 activation, which is mediated through C-terminal Grb2-SH3 and N-terminal VAV1-SH3 dimerization (59), likely influences the decrease in PLC $\gamma 1$ activation and calcium influx. VAV1 is recruited to a multiprotein complex that 
includes LAT, SLP76, and PLC $\gamma 1$ (60). PLC $\gamma 1$ hydrolyzes phosphatidylinositol 4,5-bisphosphate to produce diacylglycerol and inositol 1,4,5-triphosphate (IP3), which mediates calcium release from the endoplasmic reticulum (61). Calcium signaling induces NFAT activation, which in turn can prompt the expression of a set of anergy- and exhaustion-associated genes $(28,62,63)$. In CD28-YMFM CAR-T cells in which calcium signaling upon CAR activation is reduced, a downregulation of exhaustion pathways in comparison with wild-type CD28 CAR-T cells is observed and may be due to the reduced activation of NFAT. Strategies to eliminate the exhaustion-related gene programs downstream of NFAT activation in CAR-T cells have demonstrated enhanced antitumor activity (64). The approach undertaken here suggests that alterations to the T cell signaling program upstream of NFAT activation and calcium release may also enhance antitumor activity and preserve $\mathrm{T}$ cell function. An alternative explanation for enhanced persistence of CD28-YMFM CAR-T cells is the increased Akt activation, which may lead to higher expression of antiapoptotic proteins. Although this is an attractive hypothesis, no significant transcriptional changes of Bcl family members were observed and it remains unclear what role the enhanced Akt activation has on the persistence or antitumor activity of CD28-YMFM CAR-T cells.

The observation that CAR-T cells costimulated by ICOS were more enriched in Th17 cells than those costimulated by CD28 is well characterized as a function of ICOS signaling (65) and has been previously demonstrated in CAR-T cells through Th17-polarizing and nonpolarizing culture conditions $(19,25)$. Interestingly, GSEA analysis also demonstrated that CD28-YMFM CAR-T cells exhibited a transcriptional profile more Th17like than CD28 CAR-T cells. Th17 cells display a molecular signature consistent with stem-like properties, can give rise to Th1-like cells while also self-renewing to maintain a population of IL-17A-secreting cells, and demonstrate increased antitumor potency over Th1 cells when adoptively transferred in mouse models of melanoma $(66,67)$. In the absence of negative costimulation by CTLA4 or after anti-CTLA4 antibody blockade, $\mathrm{CD}^{+}{ }^{+} \mathrm{T}$ cells can differentiate into an $\mathrm{ICOS}^{+}$Th1-skewed population with superphysiological cytokine output capabilities (68). On a molecular level, these results suggest that the ICOS YMFM motif is at least in part responsible for $\mathrm{CD} 4^{+} \mathrm{T}$ cell differentiation toward a Th17 phenotype. It remains unclear whether the increased Akt activation, reduced activation of VAV1, or the combination of these factors in ICOS and CD28-YMFM CAR-T cells is responsible for driving Th17 differentiation. A TRAF-like motif has also been identified in ICOS and shown to be critical for the development of follicular helper T (Tfh) cells (69). This motif is not conserved in CD28-YMFM and, as a consequence, CD28-YMFM displayed an attenuated Tfh phenotype compared with CD28 CAR-T cells, suggesting that the YMFM motif does not play a role in $\mathrm{Tfh}$ development.

Achieving long-term durable persistence of CAR-T cells and associated antitumor activity will benefit patients with bulky disease and may be critical for CAR-T cell treatment success in solid tumors. This work reports a modification to a clinically relevant treatment model using a manufacturing process that is commercially validated and has the potential for rapid translation and enhancement of therapeutic efficacy. Utilization of the CD28-YMFM domain in other CAR constructs, including widely successful CD28-containing CD19 CARs, may also improve persistence and antitumor durability.

\section{Methods}

Cell lines. All cell lines were obtained from American Type Culture Collection (ATCC). The Capan-2 (pancreatic adenocarcinoma) and ASPC-1 (pancreatic adenocarcinoma ascites metastasis) cell lines were cultured in DMEM/F12. All media were supplemented with $10 \%$ fetal calf serum (FCS). All tumor cell lines were authenticated in 2016 by the University of Arizona Genetics Core and regularly validated to be free of Mycoplasma.

Isolation, transduction, and expansion of primary human $T$ lymphocytes. Blood samples were obtained from the Human Immunology Core of the University of Pennsylvania. Peripheral blood $\mathrm{CD}^{+}$and $\mathrm{CD}^{+} \mathrm{T}$ cells were negatively isolated using RosetteSep Kits (STEMCELL Technologies). Cells were cultured in RPMI 1640 media supplemented with $10 \% \mathrm{FCS}, 100 \mathrm{U} / \mathrm{mL}$ penicillin, $100 \mu \mathrm{g} / \mathrm{mL}$ streptomycin sulfate, and $10 \mathrm{mM}$ HEPES in a $37^{\circ} \mathrm{C}$ and $5 \% \mathrm{CO}_{2}$ incubator. For stimulation, $\mathrm{CD} 4^{+}$and $\mathrm{CD} 8^{+} \mathrm{T}$ cells were cultured with activating beads coated with antibodies against CD3 and CD28 at a 1:3 cell-tobead ratio. Approximately 24 hours after activation, T cells were transduced with lentiviral vectors at an MOI of 5 . For CD8 ${ }^{+} \mathrm{T}$ cells, human IL-2 (Chiron) was added every other day to a final concentration of $50 \mathrm{IU} / \mathrm{mL}$. Cells were counted and fed every 2 days and once T cells appeared to exhibit decreased growth kinetics and cell size, they were either used for functional assays or cryopreserved. All $\mathrm{T}$ cell functional assays were performed in media without cytokines.

Generation of CAR mutant constructs. CAR constructs targeting mesothelin with the SS1 scFv and costimulated by CD28, 4-1BB, and ICOS were previously described $(24,25)$. The $28 z$-YMFM mutant was generated through site-directed mutagenesis of the SS128z construct using the oligonucleotides 28z-YMFM-F (CACAGTGACTACATGTTCATGACTCCCCGCCG) and 28z-YMFM-R (CGGCGGGGAGTCATGAACATGTAGTCACTGTG). The ICOSz-YMNM mutant was generated through site-directed mutagenesis of the SS1ICOSz construct using the oligonucleotides ICOSz-YMNM-F (CTAACGGTGAATACATGAACATGAGAGCAGTGAAC) and ICOSz-YMNM-R (GTTCACTGCTCTCATGTTCATGTATTCACCGTTAG).

Western blot. T cells $\left(1 \times 10^{6}\right)$ were stimulated with magnetic beads coated with recombinant mesothelin, as previously described (19). Cell pellets were collected and frozen at indicated time points after antigen recognition. Protein lysates from cells lines were prepared in a lysis buffer containing 2\% SDS, $62.5 \mathrm{mM}$ Tris- $\mathrm{HCl}, \mathrm{pH}$ 6.8, $10 \%$ glycerol, 5\% 2-mercaptoethanol, and 0.002\% bromophenol blue. Equivalent amounts of protein were resolved by SDS-PAGE and analyzed by immunoblotting. The following primary antibodies were used: anti-phospho-Erk1/2 (p44/42) (9101S/L), anti-Erk1/2 (9107S), anti-phospho-Akt (S473) (9271), anti-Akt (2920) (Cell Signaling Technology); anti-VAV1 (sc-8039; Santa Cruz Biotechnology); anti-phospho-VAV1 (Y174) (ab76225), anti-phospho-PLC $\gamma$ (Y783) (ab76031), and anti-PLC $\gamma$ (ab41433) (Abcam). Bands were visualized by enhanced chemiluminescence (ECL). Images were captured using an Odyssey Fc system (Li-Cor Biosciences). Image processing and densitometry analysis were carried out using the Image Studio Lite software (Li-Cor Biosciences). 
Calcium measurements. Calcium influx was measured by flow cytometry as previously described (34). Briefly, human $\mathrm{CD}^{+} \mathrm{T}$ cells were electroporated with mRNA encoding CARs for SS1 $\triangle \mathrm{z}$, SS1z, SS1BBz, SS1ICOSz, SS128z, or SS128z-YMFM. Twenty-four hours after electroporation, T cells were loaded with $2 \mu \mathrm{M}$ Indo-1 AM for 30 minutes, washed, and resuspended in $\mathrm{HBSS}$ with $1 \mathrm{mM} \mathrm{Ca}^{2+}, 1 \mathrm{mM} \mathrm{Mg}^{2+}$, and $1 \%$ FBS. Loaded T cells were acquired on a BD LSRFortessa equipped with a UV laser and filters at $395 \mathrm{~nm}$ and $510 \mathrm{~nm}$ for 30 seconds to collect baseline calcium measurements. In order to measure calcium responses to CAR stimuli, cells were removed from the flow nozzle, stimulated through addition of recombinant mesothelin-Fc directly to the cell suspension, and returned to acquisition for 6 minutes. Subsequently, cells were stimulated with biotinylated OKT3 and avidin and returned to acquisition for 6 minutes to collect calcium responses to TCR stimuli. Lastly, cells were treated with $1 \mu \mathrm{g} / \mathrm{mL}$ of the calcium ionophore ionomycin to measure cellular calcium influx capability.

In vitro coculture experiments. Tumor cells $\left(1 \times 10^{5}\right)$ were seeded in 48-well plates and after 24 hours, $3 \times 10^{5} \mathrm{~T}$ cells were added (effector/target ratio $[\mathrm{E} / \mathrm{T}]=3$ ). For cytokine production assays, supernatants were collected 24 hours after coculture and analyzed using the human cytokine 30-plex panel on the Luminex system (Thermo Fisher Scientific), as previously described or assessed for human IL-2, TNF- $\alpha$, and IFN- $\gamma$ using the DuoSet ELISA Development Kit (R\&D Systems).

Flow cytometry. For all experiments, $\mathrm{T}$ cell suspensions were stained with a fixable live/dead violet stain (L34955, Life Technologies) in PBS followed by surface antibody staining in FACS buffer. The Foxp3/Transcription Factor Staining Buffer set (Life Technologies) was used for Ki67 detection. The following antibodies were used: anti-CD45-PerCp-Cy5.5 (45-9459-42), anti-CD8-APC (17-008642), anti-CD4-PE (12-0048-42), anti-TIM3-PerCPeF710 (46-310942), anti-CD25-PECy7 (25-0257-42), anti-Ki67-FITC (11-5699-42) (Life Technologies); anti-CD8-APC-H7 (560179), anti-CCR7-FITC (150503) (BD Biosciences); anti-CD4-BV510 (317444), anti-PD-1BV711 (329928) (BioLegend); anti-CD27-PECy7 (A54823), and anti-CD28-PE-CF594 (6607111) (Beckman Coulter). Expression of CAR proteins was evaluated using biotinylated goat anti-mouse IgG (115-065-072, Jackson ImmunoResearch) with streptavidin (APC or $\mathrm{PE}$ (BD Biosciences). All experiments were conducted on a BD LSRFortessa flow cytometer and analyzed with FlowJo software (Tree Star).

Mice. NSG mice were purchased from The Jackson Laboratory and bred by the Stem Cell and Xenograft Core in the vivarium at the University of Pennsylvania. The mice were housed under specific pathogen-free conditions in microisolator cages and given ad libitum access to autoclaved food and acidified water.

In vivo assessment of antimesothelin CAR-T cells. Xenograft tumors were established by subcutaneous injection of $5 \times 10^{6}$ Capan- 2 cells in the presence of a $50 \%$ solution of Matrigel (BD Biosciences) in PBS. Capan-2 tumors were allowed to grow in NSG mice for 3 weeks. Mice were then treated with 2 intravenous injections of $10 \times 10^{6} \mathrm{CAR}-\mathrm{T}$ cells (50\% chimeric receptor-positive, $1: 1 \mathrm{CD} 4^{+} / \mathrm{CD} 8^{+}$ratio) on day 0 and day 15. Tumor dimensions were measured with calipers, and tumor volumes calculated using the formula $V=0.5 \times L \times W^{2}$, where $L$ is length (longest dimension) and $W$ is width (shortest dimension). For the intraperitoneal xenograft model, $1 \times 10^{6} \mathrm{CBG}^{+}$ASPC- 1 cells were injected intraperitoneally into NSG mice in $100 \mu \mathrm{L}$ PBS. Six days after tumor inoculation, tumor bioluminescence was measured through intraperitoneal injec- tion of $3 \mathrm{mg}$ luciferin (Gold Biotechnology) and mice were randomized into treatment groups with average total flux ranging from $6.42 \times 10^{7}$ to $1.33 \times 10^{8} \mathrm{p} / \mathrm{s}$. Mice were treated the following day through an intravenous injection of $1 \times 10^{7} \mathrm{~T}$ cells, normalized to $30 \%$ CAR expression, in $100 \mu \mathrm{L}$ of PBS. Bioluminescent imaging was performed weekly in order to determine tumor burden. Peripheral blood was obtained from retro-orbital bleeding on indicated days after $\mathrm{T}$ cell injection and stained for the presence of human $\mathrm{CD} 45^{+}, \mathrm{CD}^{+}$, and $\mathrm{CD} 8^{+} \mathrm{T}$ cells. After gating on the human $\mathrm{CD} 45^{+}$population, the $\mathrm{CD} 4^{+}$and $\mathrm{CD} 8^{+}$subsets were quantified using TruCount tubes (BD Biosciences). All experiments were performed in a blinded, randomized fashion.

Ex vivo analysis of tumor-infiltrating $T$ cells. Capan-2 tumors were harvested on days 7 and 14 after $\mathrm{T}$ cell injection and processed using the gentleMACS Dissociator (Miltenyi Biotec). For flow cytometry analysis, cells were filtered to ensure a single-cell suspension and stained for viability followed by staining for murine CD45 and human CD45, EpCAM, and Ki67. To analyze the transcriptional profile of tumor-infiltrating T cells, T cells were isolated by Ficoll-Paque density gradient centrifugation (GE Healthcare). Remaining dead cells were removed using the Dead Cell Removal kit (Miltenyi Biotec) according to manufacturer's instructions.

Differential expression analysis. Frozen $\mathrm{CD}^{+} \mathrm{T}$ cells in RLT buffer were sent to BGI Genomics for RNA extraction, library preparation, and sequencing. Raw data files for in vitro stimulations found in Figure 1E were uploaded to the NCBI's Gene Expression Omnibus (GEO accession number GSE145007). FPKM and counts of ex vivo analyses found in Figure 4 and Supplemental Figure 2 are provided as Supplemental Tables 1 and 2. We removed poor quality raw reads as follows: (a) removed reads with adaptors; (b) removed reads in which unknown bases were more than $10 \%$; and (c) removed low quality reads (the percentage of low quality bases was over $50 \%$ in a read, where low quality bases were ones with sequencing quality $<5)$. After filtering, we used Bowtie2 (70) to map reads to the human reference genome, GRCh38, with the following parameters: -q --phred64 --sensitive --dpad 0 --gbar 99999999 --mp 1,1 --np 1 --score-min L,0,-0.1 -p 16 -k 200. FPKM and counts were calculated using RESM (71).

RNA-seq transcript count data were normalized using DESeq2 (72) and used to calculate differential expression between 28z, 28z-YMFM, and ICOSz CARs on day 7 and day 14, also using DESeq2. Genes were identified as significantly differentially expressed if their adjusted $P$ value was less than 0.05 and their absolute $\log _{2}$ fold change was greater than 1 (i.e., non- $\log _{2}$-transformed fold change greater than 2). Multidimensional scaling (MDS) analysis was performed on the significantly DEGs among the 3 CARs using Euclidean distances and the $\mathrm{R}$ function cmdscale() with parameters set as $\mathrm{k}=2$ dimensions, and eigen values $=$ TRUE. GSEA of immunology related gene lists from MSigDB C7 (73-75) was performed using $3 \log _{2}$ fold changeranked lists representing the expression patterns of 3 different CARs (28z-YMFM vs. $28 z$, ICOSz vs. $28 z$, ICOSz vs. $28 z-Y M N M)$. Analysis was performed using the fgsea R Bioconductor package (76) and the msigdbr R CRAN package.

Statistics. All statistical analyses were performed with Prism software version 6.0 (GraphPad). Statistically significant differences were tested by the specific tests indicated in the figure legends. The number of animals per group in each experiment was determined on the basis of previous statistical analyses by our group. Symbols indicate statistical 
significance as follows: ${ }^{*} P<0.05,{ }^{* *} P<0.01$, and ${ }^{* *} P<0.001$. A $P$ value less than 0.05 was considered significant. Where used for statistical evaluations, figure legends clarify whether ANOVA was 1-way or 2-way.

Study approval. The present studies in mice were approved by the Institutional Animal Care and Use Committee at the University of Pennsylvania, Philadelphia, Pennsylvania, USA.

\section{Author contributions}

SG and ADP designed the study, performed the experiments, analyzed and interpreted the data, and wrote the manuscript. AM analyzed the RNA-seq data. VCM performed the Western blots and analysis. CS, AW, and FL manufactured cells and performed in vivo experiments. RMY coordinated the project and edited the manuscript. $\mathrm{CHJ}$ provided conceptual guidance.

\section{Acknowledgments}

Funding from the NIH (P01CA214278, P01CA066726, and R01CA120409 to CHJ), the Department of Veterans Affairs (IK2BX004183 to ADP), and Novartis (to CHJ) supported this work. $\mathrm{CHJ}$ and ADP are members of the Parker Institute for Cancer Immunotherapy, which supported the University of Pennsylvania cancer immunotherapy program.

Address correspondence to: Avery D. Posey, Jr., 3400 Civic Center Blvd, SPE 8-106, Philadelphia, Pennsylvania 19104, USA. Phone: 773.892.4633; Email: aposey@pennmedicine.upenn.edu. Or to: Sonia Guedan, Hematology and Oncology, IDIBAPS, Rossello 149-153, Barcelona 08036, Spain. Phone: 34.93.227.5400 ext. 4528; Email: sguedan@clinic.cat.
1. Maude SL, et al. Tisagenlecleucel in children and young adults with B-cell lymphoblastic leukemia. N Engl JMed. 2018;378(5):439-448.

2. Neelapu SS, et al. Axicabtagene ciloleucel CAR T-cell therapy in refractory large B-cell lymphoma. NEnglJMed. 2017;377(26):2531-2544.

3. Schuster SJ, et al. Tisagenlecleucel in adult relapsed or refractory diffuse large B-cell lymphoma. N Engl J Med. 2019;380(1):45-56.

4. Brown CE, et al. Regression of glioblastoma after chimeric antigen receptor T-cell therapy. N EnglJ Med. 2016;375(26):2561-2569.

5. Louis CU, et al. Antitumor activity and longterm fate of chimeric antigen receptor-positive $\mathrm{T}$ cells in patients with neuroblastoma. Blood. 2011;118(23):6050-6056.

6. Ramos CA, et al. In vivo fate and activity of second- versus third-generation CD19-specific CAR-T cells in B cell non-Hodgkin's lymphomas. Mol Ther. 2018;26(12):2727-2737.

7. Porter DL, et al. Chimeric antigen receptor $\mathrm{T}$ cells persist and induce sustained remissions in relapsed refractory chronic lymphocytic leukemia. Sci Transl Med. 2015;7(303):303ra139.

8. Zolov SN, Rietberg SP, Bonifant CL. Programmed cell death protein 1 activation preferentially inhibits CD28.CAR-T cells. Cytotherapy. 2018;20(10):1259-1266.

9. Long AH, et al. 4-1BB costimulation ameliorates $\mathrm{T}$ cell exhaustion induced by tonic signaling of chimeric antigen receptors. Nat Med. 2015;21(6):581-590.

10. Gardner R, et al. Acquisition of a CD19-negative myeloid phenotype allows immune escape of MLL-rearranged B-ALL from CD19 CAR-T-cell therapy. Blood. 2016;127(20):2406-2410.

11. Sotillo E, et al. Convergence of acquired mutations and alternative splicing of CD19 enables resistance to CART-19 immunotherapy. Cancer Discov. 2015;5(12):1282-1295.

12. Orlando EJ, et al. Genetic mechanisms of target antigen loss in CAR19 therapy of acute lymphoblastic leukemia. Nat Med. 2018;24(10):1504-1506.

13. Feucht J, et al. Calibration of CAR activation potential directs alternative $T$ cell fates and therapeutic potency. Nat Med. 2019;25(1):82-88.

14. Salter AI, et al. Phosphoproteomic analysis of chimeric antigen receptor signaling reveals kinetic and quantitative differences that affect cell function. Sci Signal. 2018;11(544):eaat6753.

15. Künkele A, et al. Functional tuning of CARs reveals signaling threshold above which $\mathrm{CD} 8^{+}$ CTL antitumor potency is attenuated due to cell Fas-FasL-dependent AICD. Cancer Immunol Res. 2015;3(4):368-379.

16. Wherry EJ, Kurachi M. Molecular and cellular insights into T cell exhaustion. Nat Rev Immunol. 2015;15(8):486-499.

17. Youngblood B, Davis CW, Ahmed R. Making memories that last a lifetime: heritable functions of self-renewing memory CD8 T cells. Int Immunol. 2010;22(10):797-803.

18. Kawalekar OU, et al. Distinct signaling of coreceptors regulates specific metabolism pathways and impacts memory development in CAR T cells. Immunity. 2016;44(2):380-390.

19. Guedan S, et al. Enhancing CAR T cell persistence through ICOS and 4-1BB costimulation. JCI Insight. 2018;3(1):96976.

20. Guedan S, Calderon H, Posey AD, Maus MV Engineering and design of chimeric antigen receptors. Mol Ther Methods Clin Dev. 2019;12:145-156.

21. Zhao Z, et al. Structural design of engineered costimulation determines tumor rejection kinetics and persistence of CAR T cells. Cancer Cell. 2015;28(4):415-428.

22. Milone MC, et al. Chimeric receptors containing CD137 signal transduction domains mediate enhanced survival of $\mathrm{T}$ cells and increased antileukemic efficacy in vivo. Mol Ther. 2009;17(8):1453-1464.

23. Park JH, et al. Long-term follow-up of CD19 CAR therapy in acute lymphoblastic leukemia. $N$ Engl $J$ Med. 2018;378(5):449-459.

24. Carpenito C, et al. Control of large, established tumor xenografts with genetically retargeted human $\mathrm{T}$ cells containing CD28 and CD137 domains. Proc Natl Acad Sci U S A. 2009;106(9):3360-3365.

25. Guedan S, et al. ICOS-based chimeric antigen receptors program bipolar TH17/TH1 cells. Blood. 2014;124(7):1070-1080.

26. Harada $Y$, et al. A single amino acid alteration in cytoplasmic domain determines IL-2 promoter activation by ligation of CD28 but not inducible costimulator (ICOS). JExp Med.
2003;197(2):257-262

27. Dennehy KM, Elias F, Na SY, Fischer KD, Hünig T, Lühder F. Mitogenic CD28 signals require the exchange factor Vav1 to enhance TCR signaling at the SLP-76-Vav-Itk signalosome. J Immunol. 2007;178(3):1363-1371.

28. Martinez GJ, et al. The transcription factor NFAT promotes exhaustion of activated $\mathrm{CD}^{+} \mathrm{T}$ cells. Immunity. 2015;42(2):265-278.

29. Scott-Browne JP, et al. Dynamic changes in chromatin accessibility occur in $\mathrm{CD}^{+} \mathrm{T}$ cells responding to viral infection. Immunity. 2016;45(6):1327-1340.

30. Hassan R, Bera T, Pastan I. Mesothelin: a new target for immunotherapy. Clin Cancer Res. 2004;10(12 pt 1):3937-3942.

31. Fos C, et al. ICOS ligation recruits the p50alpha PI3K regulatory subunit to the immunological synapse. JImmunol. 2008;181(3):1969-1977.

32. Riha P, Rudd CE. CD28 co-signaling in the adaptive immune response. Self Nonself. 2010;1(3):231-240.

33. Katzav S. Vav1: an oncogene that regulates specific transcriptional activation of T cells. Blood. 2004;103(7):2443-2451.

34. Posey AD, Kawalekar OU, June CH. Measurement of intracellular ions by flow cytometry. Curr Protoc Cytom. 2015;72:9.8.1-9.8.21.

35. DeRenzo C, Gottschalk S. Genetic modification strategies to enhance CAR T cell persistence for patients with solid tumors. Front Immunol. 2019;10:218.

36. Bailey SR, et al. Human CD26 $6^{\text {high }} \mathrm{T}$ cells elicit tumor immunity against multiple malignancies via enhanced migration and persistence. Nat Commun. 2017;8(1):1961.

37. Gattinoni L, et al. A human memory T cell subset with stem cell-like properties. Nat Med. 2011;17(10):1290-1297.

38. Pule MA, et al. Virus-specific T cells engineered to coexpress tumor-specific receptors: persistence and antitumor activity in individuals with neuroblastoma. Nat Med. 2008;14(11):1264-1270.

39. Xu Y, et al. Closely related T-memory stem cells correlate with in vivo expansion of CAR.CD19-T cells and are preserved by IL-7 and IL-15. Blood. 2014;123(24):3750-3759.

40. van der Waart AB, et al. Inhibition of Akt signaling promotes the generation of superior 
tumor-reactive T cells for adoptive immunotherapy. Blood. 2014;124(23):3490-3500.

41. Guedan S, Alemany R. CAR-T cells and oncolytic viruses: joining forces to overcome the solid tumor challenge. Front Immunol. 2018;9:2460.

42. Ramakrishna S, et al. Modulation of target antigen density improves CAR T-cell functionality and persistence. Clin Cancer Res. 2019;25(17):5329-5341.

43. Ajina A, Maher J. Strategies to address chimeric antigen receptor tonic signaling. Mol Cancer Ther. 2018;17(9):1795-1815.

44. Eyquem J, et al. Targeting a CAR to the TRAC locus with CRISPR/Cas9 enhances tumour rejection. Nature. 2017;543(7643):113-117.

45. Bajgain P, et al. CAR T cell therapy for breast cancer: harnessing the tumor milieu to drive $\mathrm{T}$ cell activation. J Immunother Cancer. 2018;6(1):34.

46. Chmielewski M, Abken H. TRUCKs: the fourth generation of CARs. Expert Opin Biol Ther. 2015;15(8):1145-1154.

47. Hu B, et al. Augmentation of antitumor immunity by human and mouse CAR T cells secreting IL-18. Cell Rep. 2017;20(13):3025-3033.

48. Kagoya Y, et al. A novel chimeric antigen receptor containing a JAK-STAT signaling domain mediates superior antitumor effects. Nat Med. 2018;24(3):352-359.

49. Leen AM, et al. Reversal of tumor immune inhibition using a chimeric cytokine receptor. Mol Ther. 2014;22(6):1211-1220.

50. Cherkassky L, et al. Human CAR T cells with cell-intrinsic PD-1 checkpoint blockade resist tumor-mediated inhibition. J Clin Invest. 2016;126(8):3130-3144.

51. Liu X, et al. A chimeric switch-receptor targeting PD1 augments the efficacy of second-generation CAR T cells in advanced solid tumors. Cancer Res. 2016;76(6):1578-1590.

52. Schneider H, Cai YC, Prasad KV, Shoelson SE, Rudd CE. T cell antigen CD28 binds to the GRB-2/SOS complex, regulators of p21ras. Eur J Immunol. 1995;25(4):1044-1050.

53. Kim HH, Tharayil M, Rudd CE. Growth factor receptor-bound protein $2 \mathrm{SH} 2 / \mathrm{SH} 3$ domain binding to CD28 and its role in co-signaling. J Biol Chem. 1998;273(1):296-301.

54. Kofler DM, et al. CD28 costimulation Impairs the efficacy of a redirected t-cell antitumor attack in the presence of regulatory t cells which can be overcome by preventing Lck activation. Mol Ther. 2011;19(4):760-767.

55. Holdorf AD, et al. Proline residues in CD28 and the Src homology (SH) 3 domain of Lck are required for T cell costimulation. J Exp Med. 1999;190(3):375-384.

56. Tavano R, et al. CD28 interaction with filamin-A controls lipid raft accumulation at the T-cell immunological synapse. Nat Cell Biol. 2006;8(11):1270-1276.

57. Okkenhaug K, Rottapel R. Grb2 forms an inducible protein complex with CD28 through a Src homology 3 domain-proline interaction. JBiol Chem. 1998;273(33):21194-21202.

58. Watanabe R, et al. Grb2 and Gads exhibit different interactions with CD28 and play distinct roles in CD28-mediated costimulation. JImmunol. 2006;177(2):1085-1091.

59. Nishida M, et al. Novel recognition mode between Vav and Grb2 SH3 domains. EMBO J. 2001;20(12):2995-3007.

60. Reynolds LF, et al. Vav1 transduces T cell receptor signals to the activation of phospholipase C-gamma1 via phosphoinositide 3-kinasedependent and -independent pathways. J Exp Med. 2002;195(9):1103-1114.

61. Rhee SG. Regulation of phosphoinositidespecific phospholipase C. Annu Rev Biochem. 2001;70:281-312.

62. Macián F, García-Cózar F, Im SH, Horton HF, Byrne MC, Rao A. Transcriptional mechanisms underlying lymphocyte tolerance. Cell. 2002;109(6):719-731.

63. Mognol GP, et al. Exhaustion-associated regulatory regions in $\mathrm{CD}^{+}$tumor-infiltrating T cells. Proc Natl Acad Sci U S A. 2017;114(13):E2776-E2785.

64. Chen J, et al. NR4A transcription factors limit
CAR T cell function in solid tumours. Nature. 2019;567(7749):530-534.

65. Paulos CM, et al. The inducible costimulator (ICOS) is critical for the development of human $\mathrm{T}(\mathrm{H}) 17$ cells. Sci Transl Med. 2010;2(55):55ra78.

66. Muranski P, et al. Tumor-specific Th17-polarized cells eradicate large established melanoma. Blood. 2008;112(2):362-373.

67. Muranski P, et al. Th17 cells are long lived and retain a stem cell-like molecular signature. Immunity. 2011;35(6):972-985.

68. Wei SC, et al. Negative co-stimulation constrains $\mathrm{T}$ cell differentiation by imposing boundaries on possible cell states. Immunity. 2019;50(4):10841098.e10.

69. Pedros C, et al. A TRAF-like motif of the inducible costimulator ICOS controls development of germinal center TFH cells via the kinase TBK1. Nat Immunol. 2016;17(7):825-833.

70. Langmead B, Salzberg SL. Fast gappedread alignment with Bowtie 2. Nat Methods. 2012;9(4):357-359.

71. Li B, Dewey CN. RSEM: accurate transcript quantification from RNA-Seq data with or without a reference genome. BMC Bioinformatics. 2011;12:323.

72. Love MI, Huber W, Anders S. Moderated estimation of fold change and dispersion for RNA-seq data with DESeq2. Genome Biol. 2014;15(12):550.

73. Subramanian A, et al. Gene set enrichment analysis: a knowledge-based approach for interpreting genome-wide expression profiles. Proc Natl Acad Sci U S A. 2005;102(43):15545-15550.

74. Liberzon A, Subramanian A, Pinchback R, Thorvaldsdóttir H, Tamayo P, Mesirov JP. Molecular signatures database (MSigDB) 3.0. Bioinformatics. 2011;27(12):1739-1740.

75. Liberzon A, Birger C, Thorvaldsdóttir H, Ghandi M, Mesirov JP, Tamayo P. The Molecular Signatures Database (MSigDB) hallmark gene set collection. Cell Syst. 2015;1(6):417-425.

76. Korotkevich G, et al. Fast gene set enrichment analysis [preprint]. http://doi.org/10.1101/060012. Posted on bioRxiv October 22, 2019. 